\title{
Circulating Neutrophil Dysfunction in HBV-Related Acute-on-Chronic Liver Failure
}

OPEN ACCESS

Edited by:

Krishna Rajarathnam,

University of Texas Medical Branch at

Galveston, United States

Reviewed by:

Krzysztof Guzik,

Jagiellonian University, Poland

Katherine R. Martin,

Walter and Eliza Hall Institute of

Medical Research, Australia

*Correspondence:

Yu Shi

zjushiyu@zju.edu.cn

Zhi Chen

zjuchenzhi@zju.edu.cn

Jifang Sheng

jifang_sheng@zju.edu.cn

${ }^{t}$ These authors have contributed equally to this work and share first authorship

Specialty section: This article was submitted to Inflammation,

a section of the journal

Frontiers in Immunology

Received: 22 October 2020 Accepted: 18 January 2021

Published: 25 February 2021

Citation:

Wu W, Sun S, Wang Y, Zhao R, Ren $H$,

Li Z, Zhao H, Zhang Y, Sheng J,

Chen $Z$ and Shi $Y$ (2021) Circulating

Neutrophil Dysfunction in HBV-Related

Acute-on-Chronic Liver Failure.

Front. Immunol. 12:620365.

doi: 10.3389/fimmu.2021.620365
Wei Wu ${ }^{1 \dagger}$, Shanshan Sun ${ }^{1 \dagger}$, Yijie Wang ${ }^{1}$, Ruihong Zhao ${ }^{1}$, Haotang Ren ${ }^{1}$, Zhiwei Li ${ }^{2}$, Hong Zhao ${ }^{1}$, Yi Zhang ${ }^{3,4}$, Jifang Sheng ${ }^{1 *}$, Zhi Chen ${ }^{1 *}$ and Yu Shi ${ }^{1 *}$

1 State Key Laboratory for Diagnosis and Treatment of Infectious Diseases, National Clinical Research Center for Infectious Diseases, Collaborative Innovation Center for Diagnosis and Treatment of Infectious Diseases, The First Affiliated Hospital, Zhejiang University School of Medicine, Hangzhou, China, ${ }^{2}$ Division of Hepatobiliary and Pancreatic Surgery, Department of Surgery, First Affiliated Hospital, School of Medicine, Zhejiang University Cytometry, Hangzhou, China, ${ }^{3}$ Department of Laboratory Medicine, The First Affiliated Hospital, Zhejiang University School of Medicine, Hangzhou, China, ${ }^{4}$ Key Laboratory of Clinical In Vitro Diagnostic Techniques of Zhejiang Province, Hangzhou, China

Background and Aims: Acute-on-chronic liver failure (ACLF) is characterized by systemic inflammation accompanied by defective anti-bacterial immunity. The role of neutrophils in immune derangement of ACLF has not been fully elucidated. This study is aimed to characterize the role of circulating neutrophils in HBV-related ACLF patients.

Methods: Quantitative, phenotypic, transcriptomic, and functional alterations of circulating neutrophils were compared in ACLF and non-ACLF subjects and analyzed for associations with short-term outcomes. Interventional experiments were performed to test the impact on ACLF-patient neutrophil function in vitro.

Results: Circulating absolute neutrophil count was significantly increased in patients with ACLF and was an independent risk factor for 28-day mortality. ACLF-patient neutrophils differentially expressed a panel of surface markers (include TLR-1, TLR-2, TLR-4, CEACAM-1 and FPR1), as well as a distinct transcriptomic signature. ACLF-neutrophils displayed significantly impaired phagocytosis but an increased capacity to form neutrophil extracellular traps (NETs), which was more pronounced in patients with poor outcome. Healthy neutrophils mimicked functional characteristics of ACLF counterpart after cocultured with plasma from ACLF patients. The oxidative burst and cytokine production capacities remained unchanged. Plasma GM-CSF, IL-6, IL-8, IL-10, and IP-10 levels, as well as lipopolysaccharide (LPS) concentration, were markedly elevated in ACLF patients but not DAMP molecules HMGB-1 and HSP70. Finally, a glycolysis inhibitor, 2-deoxyglucose, reduced NET formation of ACLF patients' neutrophils.

Conclusions: Circulating ACLF-patient neutrophils exhibit alterations in number, phenotype, gene expression and function, which was associated with poor outcome and shaped by the ACLF circulatory environment. Inhibiting glycolysis can reverse neutrophil dysfunction in ACLF patients.

Keywords: hepatitis B virus, acute-on-chronic liver failure, neutrophil, phagocytosis, NET 


\section{INTRODUCTION}

Chronic hepatitis $\mathrm{B}$ virus (HBV) infection remains a major global health burden, with 350 million chronically infected individuals worldwide $(1,2)$. In addition to hepatocellular carcinoma (HCC) and decompensated cirrhosis, acute-onchronic liver failure (ACLF) is another cause of death in patients with chronic HBV infection (3). Prior studies have shown that abrupt and intensive systemic inflammation (SI) with associated immune paresis plays a key role in the pathogenesis of ACLF (4).

Neutrophils are a key component of the circulating innate immune cell population and serve as critical effector cells among the first line of defense against bacterial infections or tissue damage (5). Neutrophils are recruited to sites of inflammation by chemokines and mitochondria-derived formyl peptides, where they eliminate pathogens or remove cell debris and participate in tissue healing (5). There is increasing evidence suggesting that neutrophils play a critical role in hepatic inflammation. It has been demonstrated that both the number and ratio of circulating neutrophils are sensitive prognostic markers of the severity and outcomes of patients with cirrhosis or liver failure $(6,7)$. Neutrophil dysfunction, including impaired phagocytic activity, altered spontaneous oxidative burst, reduced complement expression, and decreased intracellular killing, is also observed in cirrhosis $(8,9)$, alcoholic hepatitis $(10)$ and acute liver failure (11). Such functional defects strongly affect mortality $(10,12,13)$. However, studies of neutrophils in ACLF are still scarce.

We previously demonstrated that the neutrophil-to-lymphocyte ratio (NLR) predicts disease progression and mortality in HBVACLF patients, consistent with other studies $(14,15)$. In this study, the phenotypic, transcriptomic, and functional alterations were characterized in circulating neutrophils from HBV-ACLF patients. And we further tested whether glycolysis is involved in functional changes of ACLF-neutrophils and its inhibition leads to reverse of functional aberrance.

\section{SUBJECTS AND METHODS}

\section{Subjects}

To confirm the absolute neutrophil count (ANC) as an independent risk factor for mortality, we first included a retrospective cohort of 667 subjects, which incorporating 63 patients with hepatitis $\mathrm{B}$ virus related acute-on-chronic liver failure (HBV-ACLF), 34 healthy controls (HC), 95 patients with compensated liver cirrhosis (CLC), and 475 patients with decompensated liver cirrhosis (DLC) admitted to the First Affiliated Hospital of Zhejiang University from May 2014 to August 2015 (cohort A). For the subsequent experiments, we prospectively enrolled a separate cohort of study subjects, including 67 patients with hepatitis B virus related acute-onchronic liver failure (HBV-ACLF), 21 healthy controls (HC) and 28 patients with compensated liver cirrhosis (CLC) from the First Affiliated Hospital of Zhejiang University between August 2018 and July 2020 (cohort B). Information of 28-day and 3month prognosis was obtained from medical records, telephone contact or a visit. The detailed individual information is shown in Table 1 and Supplementary Table 1. The diagnosis of HBVACLF was established by the criteria proposed by the Chinese Cohort on the Study of Severe Hepatitis B (COSSH) (16). HE was defined and graded by West Haven criteria (17). MELD scores were calculated according to the Malinchoc formula (18).

We excluded patients over 75 years of age and patients with confirmed or suspected malignancy, pregnancy, previous organ transplantation, severe extra-hepatic diseases, or active bacterial infections.

TABLE 1 | Baseline characteristics of study population in Cohort A.

\begin{tabular}{|c|c|c|c|c|c|c|c|}
\hline Variables & $\mathrm{HC}(\mathrm{N}=34)$ & CLC (N=95) & DLC (N=475) & ACLF (N=63) & $\begin{array}{c}\text { P value } \\
\text { (HC Vs ACLF) }\end{array}$ & $\begin{array}{c}\text { P value } \\
\text { (CLC Vs ACLF) }\end{array}$ & $\begin{array}{c}\text { P value } \\
\text { (DLC Vs ACLF) }\end{array}$ \\
\hline Age (years) & $48(18)$ & $58(17)$ & $54(14)$ & $52(20)$ & 0.395 & 0.010 & 0.512 \\
\hline Male No. (\%) & $19(56)$ & $78(82)$ & $372(78)$ & $57(91)$ & 0.000 & 0.145 & 0.024 \\
\hline HBV DNA [ $\mathrm{Lg}_{10}$ (copies/ml)] & - & $\mathrm{O}(3.9)$ & $0(4.5)$ & $3.8(5.0)$ & 0.000 & 0.001 & 0.002 \\
\hline HBeAg positivity No. (\%) & - & $27(28)$ & 136(29) & $20(31.7)$ & 0.000 & 0.718 & 0.584 \\
\hline ALT (IU/L) & $16(11)$ & $25(21)$ & 29(25) & $97(165)$ & 0.000 & 0.000 & 0.000 \\
\hline AST (IU/L) & 19(6) & 32(23) & $40(37)$ & 124(159) & 0.000 & 0.000 & 0.000 \\
\hline Albumin $(g / L)$ & $45.8 \pm 4.1$ & $38.6 \pm 4.3$ & $31.2 \pm 6.4$ & $31.1 \pm 4.9$ & 0.000 & 0.000 & 0.852 \\
\hline Serum bilirubin ( $\mu \mathrm{mol} / \mathrm{L})$ & $11(6)$ & $13(10)$ & $24(25)$ & $329(147)$ & 0.000 & 0.000 & 0.000 \\
\hline INR & $0.9(0.1)$ & $1.0(0.1)$ & $1.2(0.3)$ & $1.9(1.0)$ & 0.000 & 0.000 & 0.000 \\
\hline Creatinine $(\mu \mathrm{mol} / \mathrm{L})$ & $68(27)$ & 69(16) & $70(25)$ & 69(32) & 0.231 & 0.714 & 0.694 \\
\hline Serum sodium (mmol/L) & 142(3) & 142(3) & $141(3)$ & $137(7)$ & 0.000 & 0.000 & 0.000 \\
\hline Platelet count $\left(10^{9} / \mathrm{L}\right)$ & 193(73) & 108(83) & $64(62)$ & $73(73)$ & 0.000 & 0.001 & 0.366 \\
\hline MELD score & - & $6.4(0.7)$ & $7.2(1.5)$ & $10.5(5.4)$ & 0.000 & 0.000 & 0.000 \\
\hline Acute jaundice No. (\%) & - & $\mathrm{O}(0)$ & $50(11)$ & 63(100) & 0.000 & 0.000 & 0.000 \\
\hline Ascites No. (\%) & - & $\mathrm{O}(0)$ & $316(67)$ & $42(67)$ & 0.000 & 0.000 & 0.982 \\
\hline Hepatic encephalopathy No. (\%) & - & $\mathrm{O}(0)$ & $18(4)$ & 12(19) & 0.007 & 0.000 & 0.000 \\
\hline Upper gastrointestinal bleeding No. (\%) & - & $\mathrm{O}(0)$ & $6(1)$ & $5(8)$ & 0.093 & 0.005 & 0.000 \\
\hline 28-day transplant-free mortality No. (\%) & - & $\mathrm{O}(0)$ & $13(3)$ & $11(18)$ & 0.010 & 0.000 & 0.000 \\
\hline 3-month transplant-free mortality No. (\%) & - & $\mathrm{O}(0)$ & $22(5)$ & 19(30) & 0.000 & 0.000 & 0.000 \\
\hline
\end{tabular}

Data are expressed as mean \pm standard deviation (SD), median (interquartile range) or number (percent). ACLF, acute-on-chronic liver failure; HBV, hepatitis $B$ virus; ALT, alanine aminotransferase; AST, aspartate Aminotransferase. Comparisons between cohorts were performed by the Mann-Whitney u test or a Chi-square test. 
The study complied with the principles of the Declaration of Helsinki and was approved by the Ethics Committee of Zhejiang University. Written consent was obtained from each patient or his/her authorized representative(s).

\section{Isolation of Circulating Neutrophils}

Peripheral whole blood was drawn via venepuncture. Neutrophils were isolated using Polymorphprep (Axis-Shield PoC AS, Norway) by density centrifugation according to the manufacturer's instructions. Then neutrophils were stained using CD11b-PE (Biolegend, USA) antibodies and then analyzed by flow cytometry (Canto-II, BD, USA). The purity of neutrophils exceeded 95\% (Supplementary Figure 1). The viability of the neutrophils was examined by Countess ${ }^{\mathrm{TM}}$ II Automated Cell Counter (ThermoFisher Scientific, MA, USA) and Trypan Blue Stain (0.4\%) (ThermoFisher Scientific, MA, USA) and found to be more than $90 \%$.

\section{Analysis of Surface Markers}

After the erythrocytes were lysed, whole blood samples were stained using TLR1-PE, TLR2-AF647, TLR4-PE, CXCR1-PE, CXCR2-FITC, FPR1-APC, CD62L-PE, CD43-FITC, CD11bPE, CD64-APC (Biolegend, USA), CEACAM-1-PE (R\&D, USA), and CD16-BV510 (Sony, USA) antibodies and then analyzed by flow cytometry (Canto-II, BD, USA). Isotypematched immunoglobulins were used as negative controls.

\section{Transcriptomic Sequencing and Bioinformatic Analysis}

Total RNA was extracted from freshly isolated neutrophils using TRIzol reagent (Invitrogen, CA, USA). The sequencing library was established using an NEBNext ${ }^{\circledR}$ Ultra ${ }^{T M}$ RNA Library Prep kit for Illumina ${ }^{\circledR}$ (NEB, USA) and then sequenced on the Illumina HiSeq Xten platform according to the manufacturer's instructions.

Clean data were obtained by removing low-quality reads or reads containing adaptor/poly- $\mathrm{N}$ sequences from the raw data. Differentially expressed genes (DEGs) between groups were identified using the DESeq R package (1.10.1), and genes with an adjusted $P$ value less than 0.05 were considered differentially expressed. Gene pathway enrichment analysis of the DEGs was performed using the Reactome database; a pathway with a corrected $\mathrm{P}$ value less than 0.05 was considered significantly enriched in DEGs. All manipulations and statistical analyses were implemented using $\mathrm{R}$ freeware.

\section{Oxidative Burst Assay}

The quantification of the oxidative burst activity of neutrophils was assessed using a Phagoburst kit (Glycotope Biotechnology $\mathrm{GmbH}$, Heidelberg, Germany). Briefly, $100 \mu \mathrm{l}$ of heparinized human whole blood activated or not activated for $15 \mathrm{~min}$ at $37^{\circ} \mathrm{C}$ with E. coli. After lysis of erythrocytes, fixation and washing, cells were collected for flow cytometry. Neutrophils were gated using forward scatter (FSC) and side scatter (SSC). The percentage of cells producing reactive oxygen species (ROS) and their mean fluorescence intensity (MFI) was calculated.

\section{Phagocytosis Assay}

The phagocytosis capacity of neutrophils was assessed using a Phagotest kit (Glycotope Biotechnology GmbH, Heidelberg, Germany) according to the manufacturer's instructions. In brief, $100 \mu$ l of the anticoagulated whole blood sample was incubated with FITC-labeled E. coli bacteria for $15 \mathrm{~min}$ at $37^{\circ} \mathrm{C}$. The reaction was quenched using brilliant blue. Cells were washed, resuspended in phosphate-buffered saline (PBS) and collected for flow cytometry. Neutrophils were gated and analyzed as described above. To determine the effect of plasma on neutrophil phagocytosis, healthy human neutrophils $\left(1^{\star} 10^{6}\right)$ were isolated and cultured separately with plasma from healthy volunteers $(100 \mu \mathrm{l})$ or ACLF patients $(100 \mu \mathrm{l})$, and then the neutrophil phagocytosis was tested by the method above.

\section{Intracellular Myeloperoxidase (MPO) Assay}

One hundred microliters of the anticoagulated blood sample were incubated with E. coli $\left(3^{\star} 10^{8} / \mathrm{ml}\right)$ for $30 \mathrm{~min}$ at $37^{\circ} \mathrm{C}$. The cells were fixed and permeabilized afterwards. Then, the cells were stained with anti-MPO-FITC (MPO Flow Kit, Biolegend, USA) for $30 \mathrm{~min}$ at room temperature and washed and analyzed by flow cytometry.

\section{NET Assay}

Whole blood samples $(100 \mu \mathrm{l})$ were stimulated with opsonized $E$. Coli $\left(3^{\star} 10^{8} / \mathrm{ml}\right)$, fMLP $(0.83 \mu \mathrm{mol} / \mathrm{L})$ or PMA $(1.35 \mu \mathrm{mol} / \mathrm{L})$. An anti-human FITC-MPO antibody $(20 \mu \mathrm{l} /$ test $)$ and SYTOX Red (10 nmol/L) (Biolegend, USA) were added and the cells incubated for $30 \mathrm{~min}$ at room temperature. After lysis of the erythrocytes, the cells were analyzed using flow cytometry (Canto-II, BD, USA). Neutrophils were selected by FSC and SSC, and NET formation was defined as MPO and SYTOX Red double-positive, as previously described (19). Healthy human neutrophils were isolated $\left(1^{\star} 10^{6}\right)$, and co-cultured separately with plasma from healthy volunteers $(100 \mu \mathrm{l})$ or ACLF patients $(100 \mu \mathrm{l})$ for $3 \mathrm{~h}$ for determining the effect of plasma on neutrophil NET formation. Hereafter, the samples were stimulated with opsonized E. Coli $\left(3^{\star} 10^{8} / \mathrm{ml}\right)$ for $1 \mathrm{~h}$ and were tested the neutrophil NET production by the above method.

\section{Imaging of NET Formation}

One hundred microliters of heparinized blood in the presence or absence of E. coli $\left(3^{\star} 10^{8} / \mathrm{ml}\right)$ were incubated for $1 \mathrm{~h}$ at $37^{\circ} \mathrm{C}$. Then, an anti-human FITC-MPO antibody (20 $\mu \mathrm{l} /$ test $)$ and SYTOX Red (10 nmol/L; Biolegend, USA) were added and incubated for $30 \mathrm{~min}$ at room temperature. Images were acquired on an ImageStream Multispectral Imaging Flow Cytometer (Amnis, part of EMD Millipore, USA) using the $40 \times$ magnification objective, which provides a numerical aperture (NA) of 0.75 and a pixel dimension of $0.5 \mathrm{~m} \times 0.5 \mathrm{~m}$.

A core diameter of $7 \mu \mathrm{m}$ was used to maximize in-focus events. The $488 \mathrm{~nm}$ and $642 \mathrm{~nm}$ excitation laser were used at an output power of $150 \mathrm{~mW}$. Objects with a minimum crosssectional area of $50 \mathrm{~m}^{2}$ and a maximum of $600 \mathrm{~m}^{2}$ were collected to avoid acquiring debris or cellular aggregates. 
Typical files contained images of 20,000 cells. Cell images were analysed using IDEAS software, version 6.2. Cells in best focus were selected using the feature Brightfield (BF) Gradient RMS, a measurement of image contrast that excludes out-of-focus events. Doublets, aggregates, dead cells, and debris were excluded using SSC intensity and Syto intensity, and all analyses were restricted to single cells.

\section{Cytokine Assay}

Purified neutrophils $\left(1 \times 10^{6} / \mathrm{ml}\right)$ were stimulated with $100 \mathrm{ng} / \mathrm{ml}$ LPS (Sigma-Aldrich, Steinheim, Germany) for $4 \mathrm{~h}$ at $37^{\circ} \mathrm{C}$. In the collected supernatants, the levels of IL-2, IL-4, IL-6, IL-4, IL-10, TNF- $\alpha$ and IFN- $\gamma$ were measured using a Cytometric Bead Array (CBA) (eBioscience, USA) following the manufacturer's instructions. The lower limits of detection of the test for the cytokines were $2.6 \mathrm{pg} / \mathrm{ml}$ for IL-2 and IL-4, $3.0 \mathrm{pg} / \mathrm{ml}$ for IL-6, 2.8 $\mathrm{pg} / \mathrm{ml}$ for IL-10 and TNF- $\alpha$, and $7.1 \mathrm{pg} / \mathrm{ml}$ for IFN- $\gamma$.

The concentrations of plasma GM-CSF, MIG, IL-8, MCP-1, IP-10, G-CSF, IL- 6 and IL-10 were also determined by CBA. The lower limits of the test for the detection of the cytokines were $0.2 \mathrm{pg} / \mathrm{ml}$ for GM-CSF, $1.1 \mathrm{pg} / \mathrm{ml}$ for MIG, $1.2 \mathrm{pg} / \mathrm{ml}$ for IL-8, 1.3 $\mathrm{pg} / \mathrm{ml}$ for MCP-1, $0.5 \mathrm{pg} / \mathrm{ml}$ for IP-10, $1.6 \mathrm{pg} / \mathrm{ml}$ for G-CSF, $1.6 \mathrm{pg} / \mathrm{ml}$ for IL-6 and $0.13 \mathrm{pg} / \mathrm{ml}$ for IL- 10 .

\section{Glucose Uptake Assay}

Neutrophils $\left(1^{\star} 10^{6}\right)$ cultured in serum-free RPMI were supplemented with the fluorescent glucose analogue 2-(N-(7nitrobenzen-2-oxa-1, 3- diazol-4-yl) amino)-2-deoxyglucose (2NBDG) (Thermo Fisher Scientific, Inc.), at a final concentration of $500 \mu \mathrm{M}$. Then the neutrophils were left untreated or activated with opsonized E. Coli $\left(3^{\star} 10^{8} / \mathrm{ml}\right)$, and then incubated at $37^{\circ} \mathrm{C}$ for $4 \mathrm{~h}$; next, they were washed with PBS and assessed by flow cytometry (Canto-II, BD, USA).

\section{Glut1 Expression}

Whole blood samples $(100 \mu \mathrm{l})$ were stimulated with opsonized E. Coli $\left(3^{\star} 10^{8} / \mathrm{ml}\right)$ or left untreated for $2 \mathrm{~h}$. Then, samples were incubated with Mouse Anti-Human Glut1 PE conjugated Monoclonal Antibody (R\&D) for $30 \mathrm{~min}$ at $4^{\circ} \mathrm{C}$. After washing, cells were resuspended in PBS and assessed for Glut1 expression by flow cytometry (FACS Aria III; BD Biosciences). Isotype-matched immunoglobulins were used as negative controls.

\section{Pharmacological Inhibition of Glycolysis}

Neutrophils $\left(1^{\star} 10^{6}\right)$ were incubated in RPMI and then supplemented with 2-deoxyglucose (2-DOG) (Abmole, USA) at concentration of $2 \mathrm{~mm}$ to inhibit glycolysis for $15 \mathrm{~min}$ in a $5 \%$ CO2 atmosphere. Then, opsonized E. Coli $\left(3^{\star} 10^{8} / \mathrm{ml}\right)$ was added for another 3-h stimulation. After stimulation, cells were collected and tested for NET production as mentioned above.

\section{Endotoxin Measurement}

Blood samples were collected in pyrogen-free heparinized tubes that were dry-baked at $120^{\circ} \mathrm{C}$ for $48 \mathrm{~h}$. The plasma endotoxin level was determined by a Kinetic Turbidimetric LAL Kit for
Endotoxin Detection (Xiamen Bioendo Technology Co., Ltd, China) as previously described (20).

\section{Statistical Analysis}

All data were analysed using SPSS 20.0 for Windows (SPSS, Chicago, IL, USA), and a two-sided $\mathrm{P}$ value $<0.05$ was considered statistically significant. Continuous data were compared using One-way ANOVA (LSD method for Post-hoc analysis), Student's t-test or the Mann-Whitney u-test. Nominal variables were compared using a chi-square test. A Cox proportional hazard model was used to explore risk factors of adverse outcomes.

\section{RESULTS}

\section{Neutrophil Quantification}

In a large retrospective cohort of patients with HBV-associated cirrhosis, we demonstrated that both the absolute number of circulating neutrophils $\left(4.33 \pm 2.64^{\star} 10^{\wedge} 9 / \mathrm{L}\right)$ and the percentage $(68.66 \pm 9.85 \%)$ in patients with ACLF were significantly higher than that in patients with CLC $\left(2.48 \pm 1.29^{\star} 10^{\wedge} 9 / \mathrm{L}, 54.94 \pm\right.$ $9.68 \%$, both $\mathrm{P}<0.001)$ and DLC $\left(2.62 \pm 2.27^{\star} 10^{\wedge} 9 / \mathrm{L}, 59.26 \pm\right.$ $14.03 \%$, both $\mathrm{P}<0.001$ ) (Figures 1A, B). However, no significant differences were found between patients with early (grade 1) and advanced (grade 2-3) ACLF (Figures 1A, B). Subsequently, we found that the ANC was positively correlated with the risk of death (Figure 1C) as well as a strong risk factor associated with 28 -day short-term mortality in patients with cirrhosis [HR $(95 \%$ CI):1.23 (1.10-1.38), $\mathrm{P}<0.001)$ ], even when adjusted by age, $\mathrm{HE}$ and MELD score (Figure 1D). Additionally, there was a significant correlation between baseline neutrophil count and development of bacterial infections in patients with cirrhosis $(\mathrm{OR}=1.19,95 \% \mathrm{CI} 1.06-1.34, \mathrm{P}=0.002)$.

\section{Neutrophil Phenotypes}

The phenotypes of circulating neutrophils were compared, and a variety of surface receptors, including TLRs, chemokine receptors, adhesion molecules, and activation markers, were investigated by flow cytometry. As shown in (Table 2), we found that the expression of TLR-1, TLR-4, and CEACAM-1 on neutrophils from ACLF patients was significantly increased, but the expression of TLR-2 and FPR1 on ACLF-patient neutrophils was significantly decreased compared to that on CLC patient or HC neutrophils.

\section{Neutrophil Transcriptional Reprogramming}

The transcriptomic profiles of circulating neutrophils were compared between patients with ACLF $(\mathrm{N}=10)$, patients with CLC $(\mathrm{N}=10)$ and HC $(\mathrm{N}=10)$. Compared with CLC-neutrophils, the expression of 1022 genes were found to be upregulated in ACLF-neutrophils, and 1101 genes were downregulated (Figure 2A). And compared to HC, the expression of 726 genes were upregulated in ACLF-neutrophils, and 711 genes were downregulated (Figure 2A). 

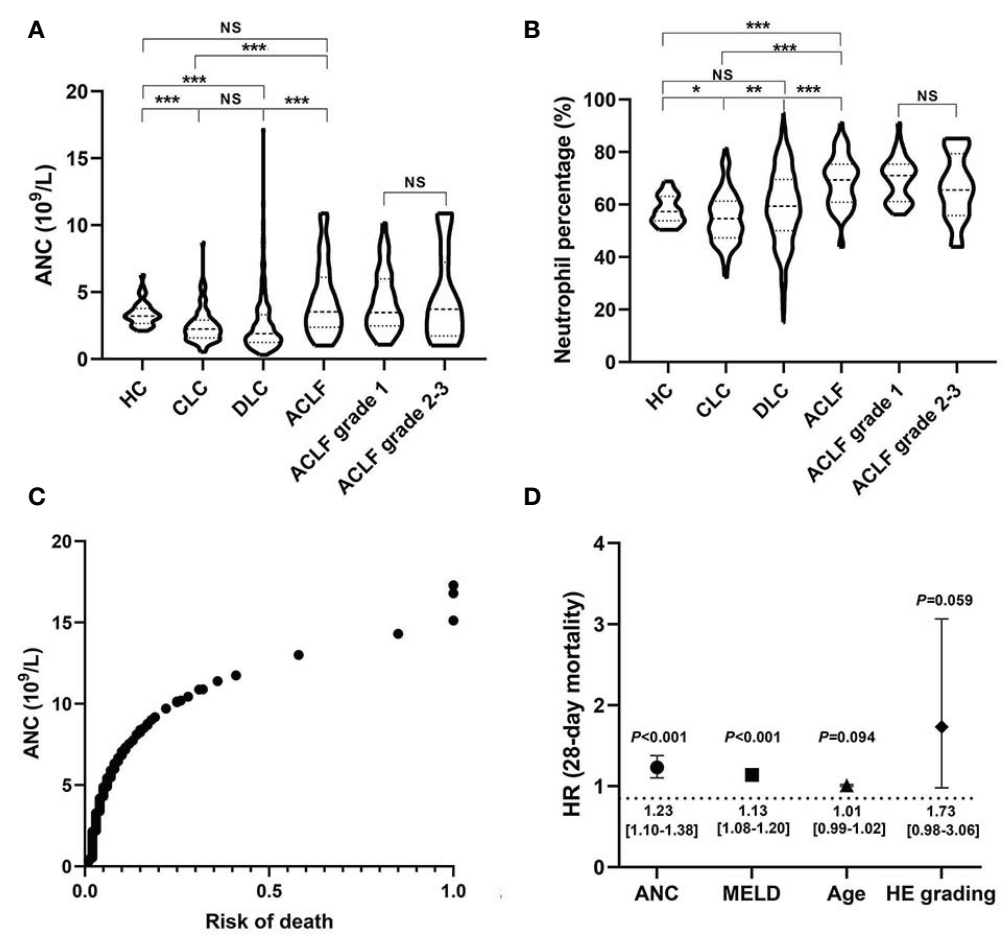

FIGURE 1 | Circulating neutrophil quantity and viability alterations in hepatitis B virus related acute-on-chronic liver failure (HBV-ACLF) patients. (A, B) Comparison of absolute counts and percentages of circulating neutrophils among patients with HBV-ACLF ( $N=63)$, decompensated liver cirrhosis ( $D L C)$ ( $N=475)$, compensated liver cirrhosis (CLC) ( $N=95)$, and healthy controls (HCs) $(\mathrm{N}=34)$, as well as the comparison between early ACLF (grade 1; $N=44)$ and advanced $A C L F$ (grade 2-3; $N=19$ ). Statistical analysis was performed by One-way ANOVA (LSD method for Post-hoc analysis) or Student $t$ test. Data are expressed as mean \pm standard deviation (SD). (C) The association between absolute neutrophil count (ANC) and 28-day risk of death in patients with HBV-related cirrhosis ( $\mathrm{N}=633$ ). Statistical analysis was performed by Cox proportional hazard model. (D) Independent 28-day risk factors for death in patients with HBV-related cirrhosis and their risk estimates with 95\% Cl. Statistical analysis was performed by the Mann-Whitney u test. Data are expressed as mean \pm standard deviation (SD). HE, Hepatic encephalopathy; MELD, Model end-stage liver disease. NS, $\mathrm{P}>0.05 ;{ }^{*} 0.01 \leq \mathrm{P}<0.05 ;{ }^{\star \star} 0.001 \leq \mathrm{P}<0.01 ;{ }^{* \star} \mathrm{P}<0.001$.

TABLE 2 | Neutrophil phenotypes in Cohort B.

\begin{tabular}{|c|c|c|c|c|c|c|}
\hline Surface markers & \multicolumn{2}{|c|}{$\mathrm{HC}(\mathrm{N}=11)$} & \multicolumn{2}{|c|}{$C-L C(\mathrm{~N}=14)$} & \multicolumn{2}{|c|}{ ACLF (N=16) } \\
\hline \multicolumn{7}{|l|}{ TLRs } \\
\hline TLR-2 & $77.0 \pm 14.9 \%$ & $785.3 \pm 256.6$ & $79.8 \pm 11.9 \%$ & $795.5 \pm 233.1$ & $68.1 \pm 11.2 \%$ *\# & $638.9 \pm 170.6^{\#}$ \\
\hline TLR-4 & $29.5 \pm 12.8 \%$ & $275.6 \pm 69.2$ & $24.9 \pm 12.6 \%$ & $255.6 \pm 72.9$ & $35.2 \pm 11.6 \%^{\#}$ & $296.1 \pm 63.2$ \\
\hline \multicolumn{7}{|c|}{ Chemokine Receptors } \\
\hline FPR1 & $82.3 \pm 14.1 \%$ & $1,3634 \pm 5,241$ & $91.9 \pm 9.4 \% *$ & $13,803 \pm 4,618$ & $73.5 \pm 24.2 \%^{\#}$ & $1,0215 \pm 6,030$ \\
\hline \multicolumn{7}{|l|}{ Adhesion receptors } \\
\hline CD62L & $67.5 \pm 20.0 \%$ & $1,114.1 \pm 525.1$ & $72.3 \pm 19.7 \%$ & $2,496.1 \pm 4,472.7$ & $71.1 \pm 22.9 \%$ & $2,010.1 \pm 1,757.8$ \\
\hline CD43 & $81.6 \pm 21.9 \%$ & $3,330.3 \pm 1,020.8$ & $78.9 \pm 23.7 \%$ & $2,847.6 \pm 791.5$ & $82.1 \pm 12.8 \%$ & $2,765.7 \pm 700.9$ \\
\hline CD11b & $99.9 \pm 0.06 \%$ & $13,322 \pm 7,368$ & $99.8 \pm 0.2 \%$ & $1,1252 \pm 4,523$ & $99.7 \pm 0.8 \%$ & $12,555 \pm 4,381$ \\
\hline CEACAM-1 & $40.4 \pm 27.6 \%$ & $528.4 \pm 282.4$ & $29.6 \pm 15.1 \%$ & $403.7 \pm 138.3$ & $54.0 \pm 23.4 \%^{\#}$ & $673.7 \pm 320.3^{\#}$ \\
\hline
\end{tabular}

${ }^{\#}$ represents a significant difference $(P<0.05)$ between patients with $A C L F$ and $C L C$; ${ }^{*}$ represents a significant difference $(P<0.05)$ between patients with $A C L F$ and $H C$. * ${ }^{*}$ represents a significant difference $(P<0.05)$ between patients with CLC and HC. Comparisons between groups were performed by One-way ANOVA (LSD method for Post-hoc analysis). Data are expressed as mean \pm standard deviation (SD). 


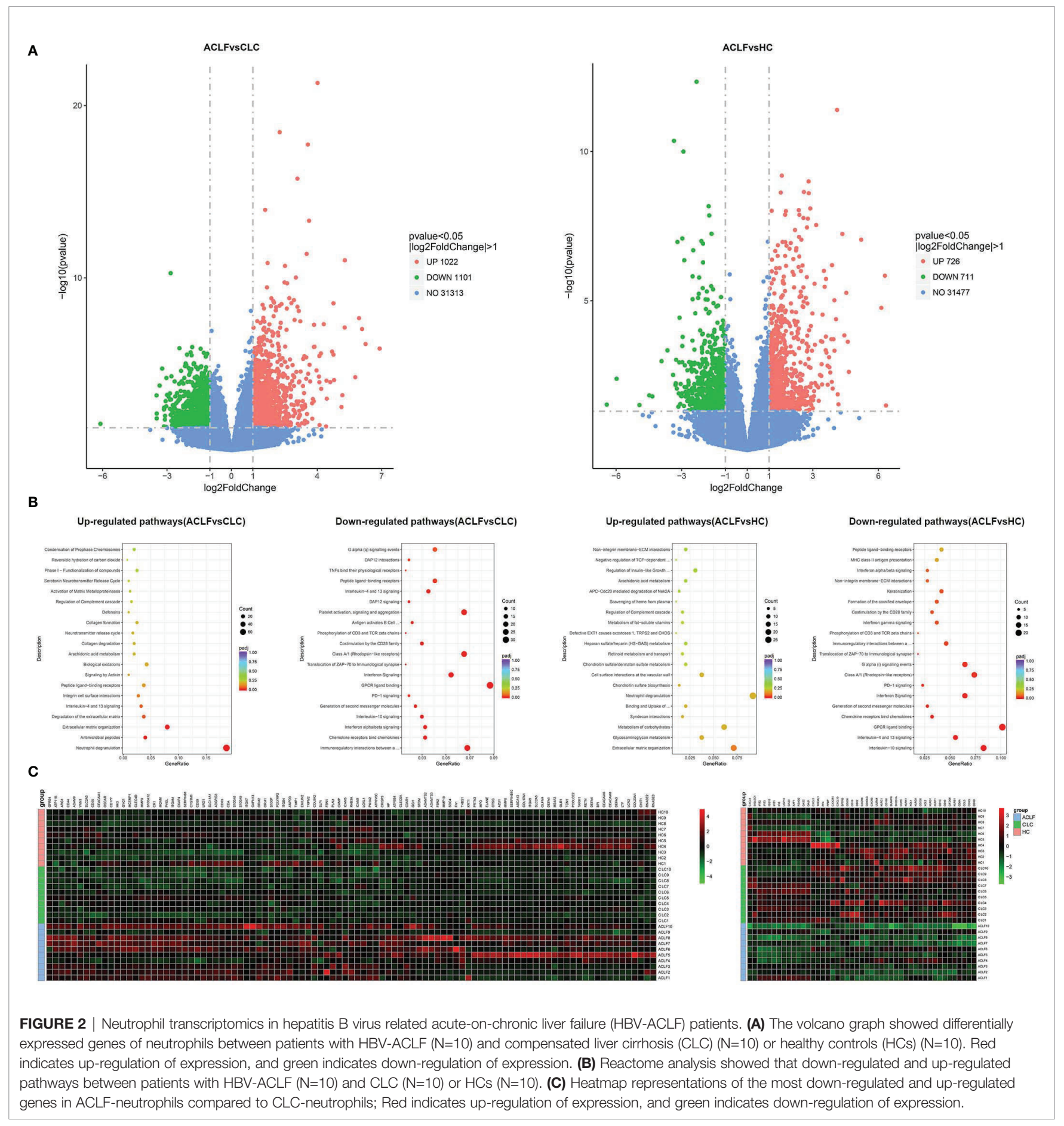

The pathway analysis identified multiple pathways enriched or down-regulated in ACLF-neutrophils when comparing with neutrophils from CLC or HC groups. For example, the pathway analysis identified multiple pathways enriched or down-regulated in ACLF-neutrophils when comparing with neutrophils from CLC or HC groups, e.g., genes associated with degranulation (representative genes such as myeloperoxidase, cathepsin G and elastase), antibacterial immunity (representative genes such as defensin alpha 1 and defensin alpha 4), and extracellular matrix remodeling (representative genes such as matrix metallopeptidase 8 and matrix metallopeptidase 9) were significantly enriched in neutrophils from ACLF patients in comparison with CLCneutrophils (Figures 2B, C). In contrast, the expression of genes associated with interactions with adaptive immunity (representative genes such as killer cell lectin-like receptor G1 and cytotoxic and regulatory $\mathrm{T}$ cell molecule), chemokine production (representative genes such as $\mathrm{C}-\mathrm{X} 3-\mathrm{C}$ motif chemokine receptor $1, \mathrm{C}-\mathrm{X}-\mathrm{C}$ motif chemokine ligand 8 and $\mathrm{C}$ - 
$\mathrm{C}$ motif chemokine receptor 5) and the IFN $\alpha / \beta$ pathway (representative genes such as interferon-induced protein with tetratricopeptide repeats 3 , interferon-induced protein with tetratricopeptide repeats 1 and interferon alpha-inducible protein 6) was significantly downregulated (Figures 2B, C).

\section{Neutrophil Functional Aberrance}

Next, we tested whether multiple neutrophil functions, including oxidative burst, phagocytosis, degranulation, NET formation and cytokine production, were altered in ACLF patients. After stimulation with E. coli, a marked stepwise decrease in phagocytosis was observed across the three groups (percentage: HC: $68.61 \pm 24.55 \%$, CLC: $50.79 \pm 18.81 \%$, ACLF: $26.22 \pm$ 17.93\%; MFI: HC: $599.38 \pm 281.64$, CLC: $466.21 \pm 264.54$, ACLF: $175.33 \pm 134.38$ : Figures 3B, D). In contrast, the stepwise increase in NET formation in response to either E. coli, fMLP or PMA showed a sharp increase in ACLF patients (percentage after E. coli stimulation: $13.34 \pm 10.92 \%$; Figures 3F, H). The morphological features of NET-forming neutrophils are shown in Figure 3I.

No significant differences in oxidative burst capacity were found between the ACLF and CLC or HC groups, except for a moderate increase in spontaneous oxidative burst in ACLF patients compared to $\mathrm{HC}(\mathrm{P}=0.019$; Figures $\mathbf{3 A}, \mathbf{C})$. Then we found a significantly increased intracellular MPO level, as indicated by either percentage (ACLF vs CLC, $\mathrm{P}=0$ ) or MFI ( $\mathrm{ACLF}$ vs CLC, $\mathrm{P}=0$ ), in ACLF patients compared to patients with CLC but not compared to HC (Figure 3E), which suggests changes of neutrophil degranulation function during disease development, but further experiments and exploration are still needed to determine this. In addition, the ratio of percent of NET formation to phagocytosis was significantly higher in deceased ACLF patients than survivors (deceased at 28-day: $1.00 \pm 0.03$, survivors at 28 -day: $0.23 \pm 0.06, \mathrm{P}=0.027$, Survival at 3 -month: $0.09 \pm 0.03$, Death at 3-month: $0.19 \pm 0.05, P=0.024$ : Figure 3G). After LPS stimulation, neutrophils produced detectable but low levels of cytokines such as IL-2, IL-4, IL-6, IL-10, TNF- $\alpha$, and IFN- $\gamma$, and no significant differences were found among the groups (Figure 3J).

\section{Circulation Environment}

We hypothesized the circulating neutrophil dysfunction in ACLF patients may result from their distinct circulatory environment. The plasma co-culture experiments revealed that healthy neutrophils became less phagocytic (percentage: HC plasma: $77.28 \pm 14.08 \%$, ACLF plasma: $5.9 \pm 3.21 \%, \mathrm{P}=0$; MFI: HC plasma: $1581 \pm 638.66$, ACLF plasma: $99.8 \pm 43.25, \mathrm{P}=0.043$ : Figure 4A), but produced more NET (percentage: HC plasma: $0.36 \pm 0.26 \%$, ACLF plasma: $10.8 \pm 8.22 \%, P=0.009$ : Figure $4 B$ ) in stimulation to E. coli after incubation with ACLF plasma, a functional phenotype mimicking ACLF-neutrophils. Then, the components of circulatory environment underlying neutrophil dysfunction was investigated. Levels of inflammatory mediators, including GM-CSF, IL-6, IL-8, IL-10, and IP-10, were significantly increased in ACLF patients (Figure 4C). Additionally, the concentration of the bacterial product LPS was markedly increased in the circulation of patients with
ACLF (Figure 4D). However, elevated concentrations of the DAMP molecules HMGB-1 and HSP were not seen in ACLF patients (Figure 4E).

\section{The Effect of Glycolysis Inhibition on NET Formation}

A recent study has shown that glycolysis is required for NET formation in healthy neutrophils and therefore we tested whether inhibiting glycolysis is able to correct over-

production of NET in ACLF-patient neutrophils. After E. coli stimulation, glucose uptake and transport (as indicated by Glut1 expression) were markedly enhanced in neutrophils from either HC, CLC, or ACLF patients (Figures 5A, B). 2-DOG, an inhibitor of glycolysis, significantly reduced NET formation of neutrophils either in patients with ACLF (Percentage of NETproducing neutrophils: $1.81 \pm 1.84 \%$ vs $1.11 \pm 1.61 \%, \mathrm{P}=0.012)$, CLC ( $1.93 \pm 0.90 \%$ vs $1.61 \pm 0.58 \%, \mathrm{P}=0.042)$, or controls $(1.57 \pm$ $1.47 \%$ vs $0.74 \pm 0.93 \%, \mathrm{P}=0.028$ ) (Figure $5 \mathrm{C}$ ).

\section{DISCUSSION}

Neutrophils are key effector cells of host innate immunity and play a critical role in resisting bacterial infections and dealing with tissue damage (21). HBV-ACLF is an innate immunemediated and systemic inflammation-driven syndrome characterized by multi-organ failure/dysfunction and is frequently complicated by bacterial infection $(3,22)$. The present study revealed diverse alterations in the quantity, phenotype, transcription, and function of neutrophils from ACLF patients. The findings of this study suggest that the immunological feature of ACLF is intensive immune system activation with concurrent immune paresis.

The most remarkable functional aberrance of ACLF-patient neutrophils was a significant decrease in phagocytic activity but an increase in NET formation in response to bacterial stimulation. Neutrophils kill microbes via phagocytosis, oxidant species generation, and cell proteolytic machinery activation (23). The formation of NETs represents an alternative mechanism to defend against invading pathogens. The formation and release of sticky web-like structures composed of decondensed DNA decorated with citrullinated histone and neutrophil granule proteins enable neutrophils to catch and kill pathogens (24). We hypothesize that the increase in NET formation is a compensatory mechanism for the impaired phagocytic capacity. When phagocytosis is weakened or ineffective, the release of NETs prevents microbes from escaping immunity (25).

The neutrophil transcriptomics demonstrated several major transcriptional reprogramming signatures of ACLF-patient neutrophils. The active transcription of degranulation-related genes concurs with the increased propensity of neutrophils to produce NETs, the formation of which requires neutrophil granule proteins, such as MPO and neutrophil elastase. Second, the enrichment of ECM-related genes suggests the role of neutrophils in tissue repair. In contrast, the expression of 
A

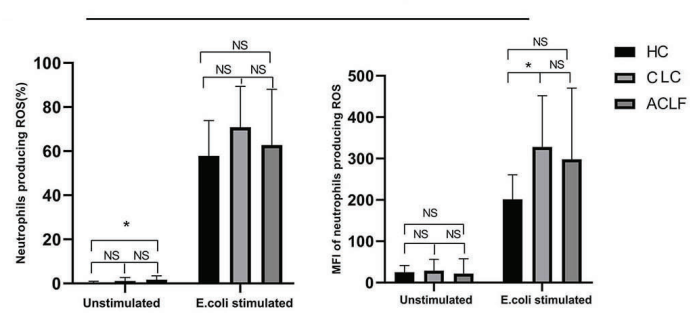

Phagocytosis of E.coli

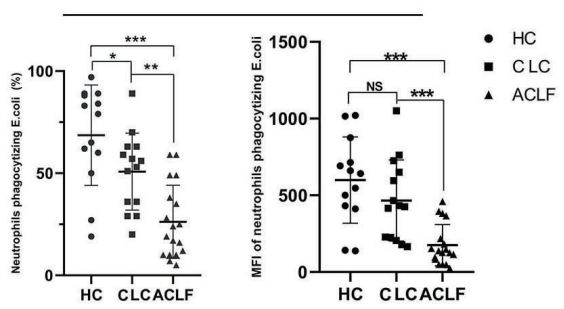

C

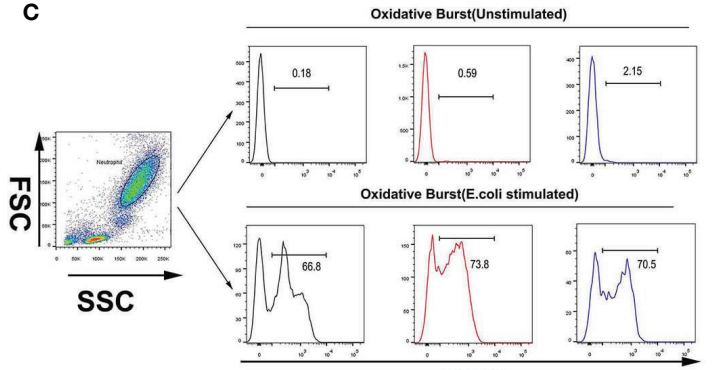

$\mathbf{E}$

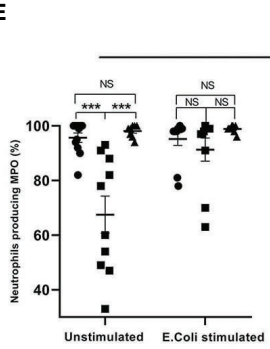

MPO

FITC

- CLC

- ACLF

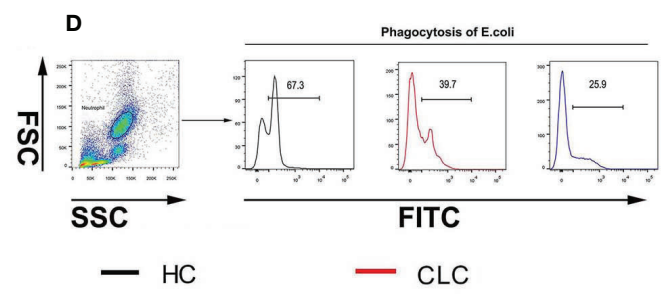

ACLF

H

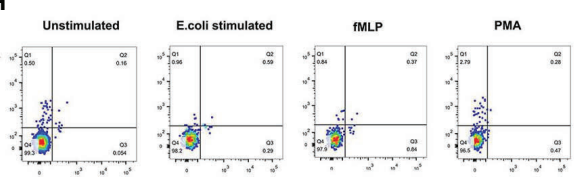

CLC 离
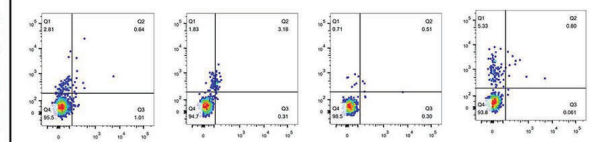

F

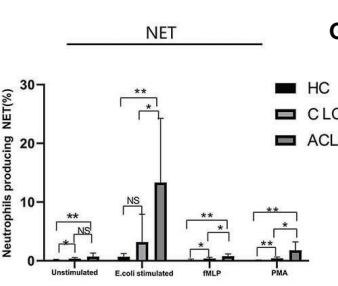

G

E.coli stimulated

D Survivor

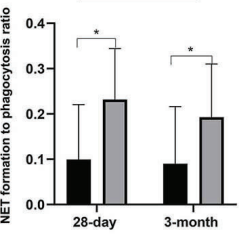

ACLF

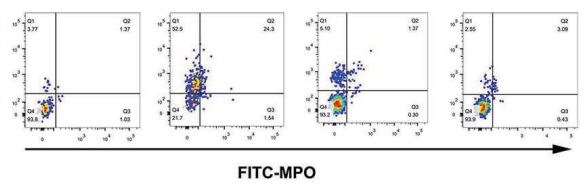

I

Blank

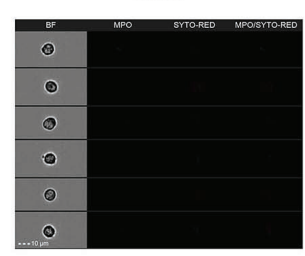

Unstimulated

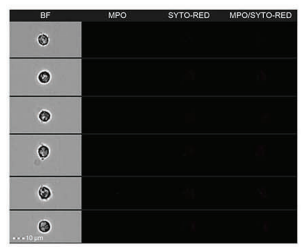

NET Positive Cell

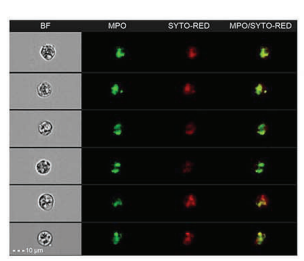

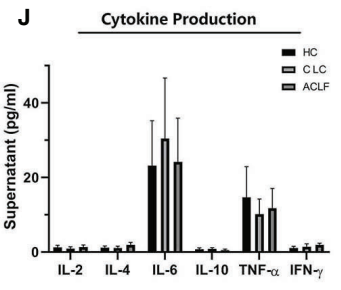

FIGURE 3 | Multiple neutrophils functions in hepatitis B virus related acute-on-chronic liver failure (HBV-ACLF) patients. (A, C) The percentage and mean fluorescence intensity (MFI) of neutrophils producing reactive oxygen metabolites with or without E.coli stimulation in patients with $\mathrm{HBV}$-ACLF ( $\mathrm{N}=18$ ), compensated liver cirrhosis $(\mathrm{CLC})(\mathrm{N}=14)$ or healthy control $(\mathrm{HC})(\mathrm{N}=13)$ and the representative graph showing the gating strategy, Data are expressed as mean \pm standard deviation (SD). (B, D) The percentage and MFI of neutrophils phagocytizing E.coli in patients with HBV-ACLF ( $N=18), C L C(N=14)$, and $H C$ ( $N=13)$ and the representative graph showing the gating strategy, Data are expressed as mean \pm standard deviation (SD). (E) The percentage and MFI of neutrophils producing intracellular myeloperoxidase (MPO) in patients with HBV-ACLF ( $\mathrm{N}=8)$, CLC ( $\mathrm{N}=10)$, or $\mathrm{HC}(\mathrm{N}=11)$. Data are expressed as mean \pm standard error of mean (SEM). $(\mathbf{F}, \mathbf{H})$ The percentage of neutrophils producing NET in patients with HBV-ACLF $(\mathrm{N}=7), \mathrm{CLC}(\mathrm{N}=6)$, and $\mathrm{HC}(\mathrm{N}=8)$ and the representative graph showing the gating strategy, Data are expressed as mean \pm standard deviation (SD). (G) The ratio of percent of neutrophil extracellular trap (NET) producing neutrophils to that of phagocytic neutrophils in deceased ACLF patients or survivors at 28-day (deaths: four cases, survivors: 18 cases) or 3-month (deaths: six cases, survivors: 15cases). Data are expressed as mean \pm standard deviation (SD). (I) Imaging flow of NET formation. The four columns showed the morphology, MPO fluorescence, SYTOX RED fluorescence and MPO-SYTOX RED merged fluorescence of single selected neutrophil. (J) Supernatant levels of IL-2, IL-4, IL-6, IL-10, TNF- $\alpha$, and IFN$\gamma$ produced by neutrophils from patients with HBV-ACLF (N=7), CLC ( $N=5)$, and HC (N=6) after stimulated by lipopolysaccharide (LPS). Data are expressed as mean \pm standard error of mean (SEM). Statistical analysis was performed by the Mann-Whitney $u$ test. NS, $\mathrm{P}>0.05 ;{ }^{*} 0.01 \leq \mathrm{P}<0.05 ;{ }^{\star \star} 0.001 \leq \mathrm{P}<0.01 ;{ }^{\star \star \star} \mathrm{P}<0.001$. 
A

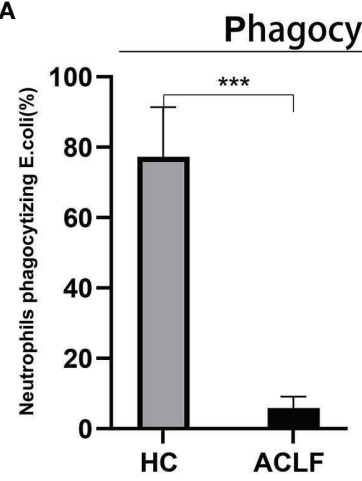

C

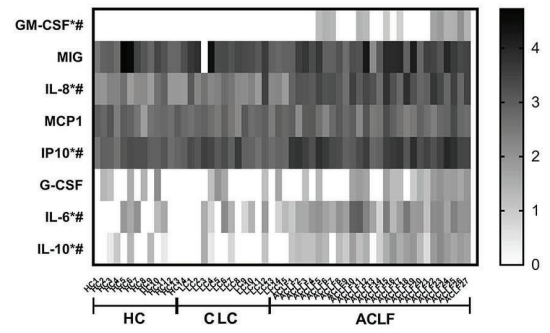

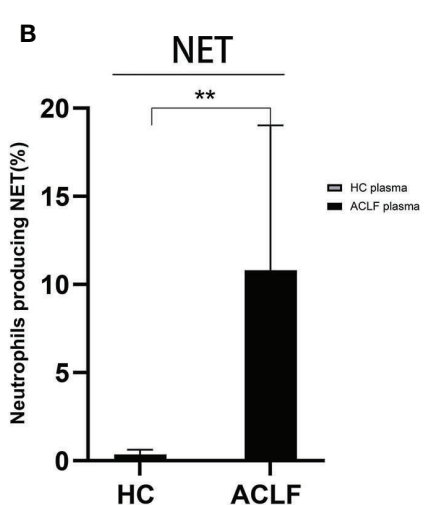

E
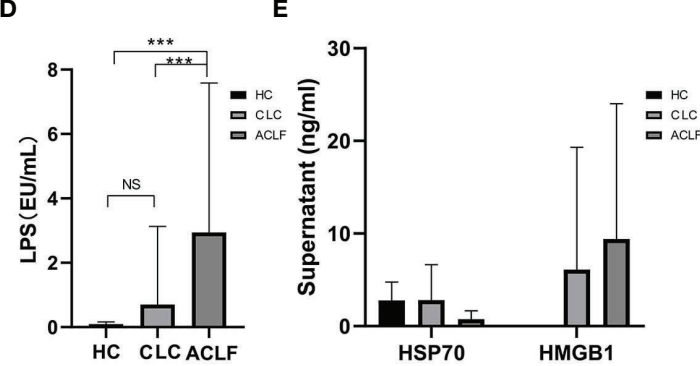

FIGURE 4 | Circulation environment in hepatitis B virus related acute-on-chronic liver failure (HBV-ACLF) patients. (A) The percentage and mean fluorescence intensity (MFI) of neutrophils phagocytizing E.coli of healthy controls incubated with the plasma of $A C L F(N=5)$ and healthy control (HC) ( $N=5)$. (B) The percentage of neutrophils producing neutrophil extracellular trap (NET) of healthy controls incubated with the plasma of ACLF (N=5) and $\mathrm{HC}$ ( $\mathrm{N}=5)$. (C) Comparison of plasma levels of inflammatory mediators GM-CSF, MIG, IL-8, MCP-1, IP-10, G-CSF, IL-6, and IL-10 among patients with HBV-ACLF (N=27), CLC (N=15), or HC (N=14). * Represents a significant difference $(P<0.05)$ between patients with ACLF and CLC; \# represents a significant difference $(P<0.05)$ between patients with ACLF and HC. (D) Comparison of plasma lipopolysaccharide (LPS) among patients with HBV-ACLF ( $N=22)$, CLC ( $N=18)$, or HC ( $N=16)$. (E) Comparison of DAMP molecules HMGB-1 and HSP among patients with HBV-ACLF $(\mathrm{N}=6)$, CLC $(\mathrm{N}=9)$, or $\mathrm{HC}(\mathrm{N}=8)$. Data are expressed as mean \pm standard deviation (SD). Statistical analysis was performed by the Mann-Whitney $\mathrm{u}$ test. NS, $\mathrm{P}>0.05 ;{ }^{*} 0.01 \leq \mathrm{P}<0.05$; ${ }^{\star *} 0.001 \leq \mathrm{P}<0.01$; ${ }^{* \star *} \mathrm{P}<0.001$.

A

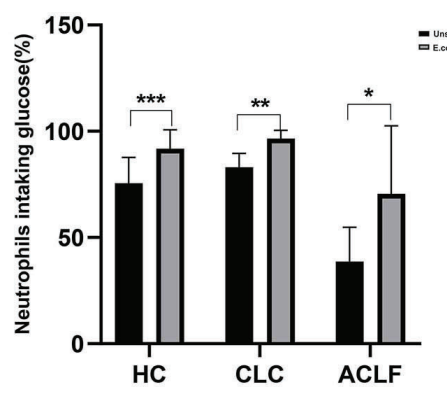

B

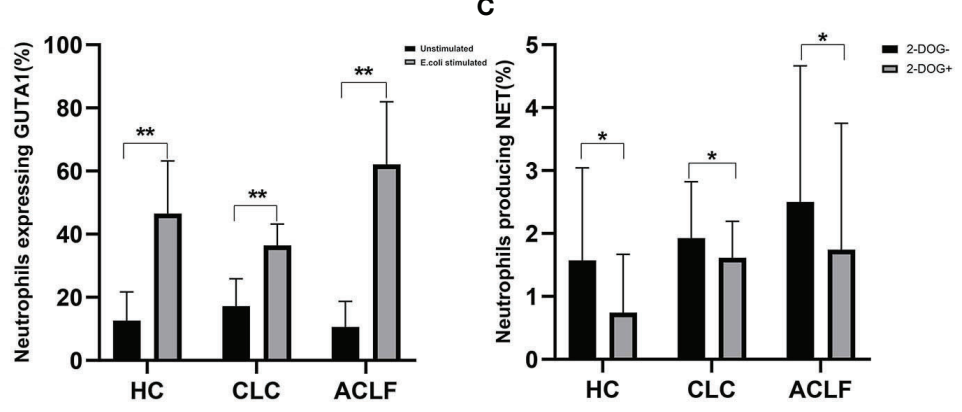

FIGURE 5 | The effect of glycolysis inhibition on neutrophil extracellular trap (NET) formation. (A) The percentage of neutrophils uptaking glucose in patients with hepatitis $B$ virus related acute-on-chronic liver failure (HBV-ACLF) ( $N=5)$, compensated liver cirrhosis $(C L C)(N=6)$, and healthy controls $(H C s)(N=8)$ following $E$. coli stimulation. (B) The percentage of neutrophils expressing Glut1 in patients with HBV-ACLF ( $N=5)$, CLC (N=7), and HCs (N=6) following E. coli stimulation. (C) After additional supplement of 2-DOG, the percentage of neutrophils producing NET was determined from patients with $\mathrm{HBV}$ - $\mathrm{ACLF}(\mathrm{N}=9)$, $\mathrm{CLC}(\mathrm{N}=7)$, and $\mathrm{HCs}(\mathrm{N}=7)$. Data are expressed as mean \pm standard deviation (SD). Statistical analysis was performed by the Mann-Whitney $\mathrm{u}$ test. NS, $\mathrm{P}>0.05 ;{ }^{\star} 0.01 \leq \mathrm{P}<0.05$; ${ }^{* \star} 0.001 \leq$ $\mathrm{P}<0.01 ;{ }^{\star \star \star} \mathrm{P}<0.001$

genes associated with interactions with adaptive immunity was significantly suppressed. As the formation of NET in neutrophils is elevated in ACLF patients but not those with CLC, we hypothesize neutrophil functional reprogramming may be shaped by the circulatory microenvironment in ACLF, as confirmed by the incubation experiment showing that healthy neutrophils acquired a functional phenotype like neutrophils from ACLF patients after co-culture with ACLF plasma. It is not 
clear, however, the specific components in ACLF plasma contribute to neutrophil dysfunction. The prevailing hypothesis is that systemic innate immune cells are activated by pathogenassociated molecular patterns (PAMPs) produced and released from the leaking intestinal tract, as well as by DAMPs released from necrotic hepatocytes through pattern recognition receptors, such as TLRs and nucleotide-binding oligomerization domainlike receptors (NLRs) (26). In our study, however, the levels of DAMP molecules, such as HMGB-1 and HSP70, were not significantly elevated. Nevertheless, the role of DAMPs in inducing neutrophil activation cannot be excluded. The release of DAMPs into the circulation may occur at disease onset and would not be significant at later stages. Some DAMPs are insoluble but exposed on the cell surface. For example, HSPs may translocate to the cell surface after atypical cell death (27). Other DAMP molecules should be investigated. In contrast, the level of LPS was found to be significantly increased in the systemic circulation of HBV-ACLF patients. Our prior study also showed an increase in circulating bacterial DNA in HBVACLF patients (28). Furthermore, previous studies have shown that the effect of ACLF plasma on neutrophil phagocytic activity and resting oxidative stress is significantly attenuated after antibiotic treatment (10). All these findings suggest that persistent stimulation by PAMPs plays an important role in inducing neutrophil functional alterations in ACLF. In addition, the spill-over of inflammatory mediators into the circulation of ACLF patients may contribute to neutrophil functional alterations. IL-6 and IL-8, the levels of which were significantly elevated in the plasma of patients with ACLF, are reported to prime resting neutrophils (29). Therefore, a combination of inflammatory and microbial signals may cause neutrophil functional reprogramming in HBV-ACLF patients. Likewise, microbial signals may have impact on the neutrophil phenotypes. The increased expression of TLR-1 and TLR-4 may be associated with the neutrophil response to recognize enrichment of specific bacterial products such as LPS or bacterial lipoproteins and LTAs in circulation of HBV-ACLF patients. And subsequent TLR activation is reported to regulate chemokine receptor expression (30).

Functional changes in neutrophils have potential clinical implications. Previous studies have shown that impaired neutrophil phagocytosis is closely associated with an increased risk of bacterial infection in patients with cirrhosis or alcoholic hepatitis (10). In this study, a severe decline in phagocytic activity was found in ACLF-patient neutrophils. The functional defects of neutrophils are consistent with the increased susceptibility of ACLF patients to bacterial infection and the increased shortterm mortality (31). At the same time, we found that neutrophil count was positively correlated with the risk of short-term death. It is well known that Granulocyte-colony stimulating factor (G-CSF) can expand neutrophil populations while improve the survival of ACLF patients at the same time $(32,33)$. These conclusions seem to be mutually contradictory, but in view of a previous study proving that G-CSF can reverse neutrophil phagocytosis, the contradiction can be partly explained by the dual influences of G-CSF on ACLF patient survival ratio (34). In addition, excessive
NET formation may cause collateral immune damage (35-37). In contrast, the use of Granulocyte-colony stimulating factor (GCSF), was reported to improve the survival of ACLF patients and the clinical benefit may be partially attributed to the effect of reversing neutrophil phagocytosis by G-CSF (32-34). Excessive NET formation may clog the pancreatic duct and induce aseptic inflammation in acute pancreatitis (38). NETs may promote thrombosis, and thus leading to microcirculatory disorders (39). Furthermore, NET components can stimulate monocytes/ macrophages and amplify the inflammatory response (40). Therefore, skewed neutrophil function may be a hallmark feature of ACLF-associated immune dysfunction phenotypes, along with concurrent SI and immunosuppression, which predisposes ACLF patients to inflammation-related organ damage and an increased risk of bacterial infection. Our preliminary data has shown that the severity of neutrophil dysfunction, as indicated by the ratio of NET-producing cells to phagocytic cells upon E. coli challenge, correlated with the shortterm outcome of ACLF patients. Nevertheless, further large, prospective studies are needed to validate the association.

It has been shown that innate immune cell function can be controlled at the metabolic level. A recent study has shown that glucose metabolism is actively involved in NET formation in healthy neutrophils (41). Concurrent with this study, we demonstrate here that glucose uptake and transport were activated both in health, CLC and ACLF neutrophils following stimulation, Further, blocking glycolysis using an inhibitor can reduce the capacity to produce NET in neutrophil derived from all the three groups. This finding suggested a markedly increase of glucose requirement for NET formation and the potential of metabolic rewiring of neutrophils in modifying the immunological function in ACLF patients.

In conclusion, circulating neutrophils from HBV-ACLF patients present quantitative, phenotypic, transcriptional, and functional alterations. The functional alterations are shaped by the specific ACLF circulatory microenvironment and associated with poor outcome. Further large studies should be performed to investigate the association between neutrophil dysfunction and disease progression, complications, and mortality. Additionally, the efficacy and safety of therapies targeting at glycolysis for reversing neutrophil dysfunction in ACLF merits further investigation.

\section{DATA AVAILABILITY STATEMENT}

The original contributions presented in the study are included in the article/Supplementary Material. Further inquiries can be directed to the corresponding authors.

\section{ETHICS STATEMENT}

The study received approval from the Ethics committee of The First Affiliated Hospital of Zhejiang University (2017-421). The 
patients/participants provided their written informed consent to participate in this study.

\section{AUTHOR CONTRIBUTIONS}

YS, ZC, and JS conceived the study. WW and SS conceived and designed the experiments while WW, SS, YW, RZ, and HR performed them. YW, ZL, HZ, and YZ were involved in clinical data collection. YS, WW, SS, and YW wrote the manuscript. All authors contributed to the article and approved the submitted version.

\section{FUNDING}

This research was funded by the National Natural Science Foundation of China (grant nos. 81671949, 81870425, and

\section{REFERENCES}

1. Shi Y, Zheng M. Hepatitis B virus persistence and reactivation. BMJ (2020) 370:m2200. doi: 10.1136/bmj.m2200

2. Wang FS, Fan JG, Zhang Z, Gao B, Wang HY. The global burden of liver disease: the major impact of China. Hepatology (2014) 60(6):2099-108. doi: 10.1002/hep.27406

3. Shi Y, Yang Y, Hu Y, Wu W, Yang Q, Zheng M, et al. Acute-on-chronic liver failure precipitated by hepatic injury is distinct from that precipitated by extrahepatic insults. Hepatology (2015) 62(1):232-42. doi: 10.1002/hep.27795

4. Jalan R, Gines P, Olson JC, Mookerjee RP, Moreau R, Garcia-Tsao G, et al. Acute-on chronic liver failure. J Hepatol (2012) 57(6):1336-48. doi: 10.1016/ j.jhep.2012.06.026

5. Kolaczkowska E, Kubes P. Neutrophil recruitment and function in health and inflammation. Nat Rev Immunol (2013) 13(3):159-75. doi: 10.1038/nri3399

6. Rice J, Dodge JL, Bambha KM, Bajaj JS, Reddy KR, Gralla J, et al. Neutrophilto-Lymphocyte Ratio Associates Independently With Mortality in Hospitalized Patients With Cirrhosis. Clin Gastroenterol Hepatol (2018) 16 (11):1786-1791 el. doi: 10.1016/j.cgh.2018.04.045

7. Khanam A, Trehanpati N, Riese P, Rastogi A, Guzman CA, Sarin SK. Blockade of Neutrophil's Chemokine Receptors CXCR1/2 Abrogate Liver Damage in Acute-on-Chronic Liver Failure. Front Immunol (2017) 8:464. doi: 10.3389/fimmu.2017.00464

8. Tritto G, Bechlis Z, Stadlbauer V, Davies N, Frances R, Shah N, et al. Evidence of neutrophil functional defect despite inflammation in stable cirrhosis. J Hepatol (2011) 55(3):574-81. doi: 10.1016/j.jhep.2010.11.034

9. Boussif A, Rolas L, Weiss E, Bouriche H, Moreau R, Perianin A. Impaired intracellular signaling, myeloperoxidase release and bactericidal activity of neutrophils from patients with alcoholic cirrhosis. J Hepatol (2016) 64 (5):1041-8. doi: 10.1016/j.jhep.2015.12.005

10. Mookerjee RP, Stadlbauer V, Lidder S, Wright GA, Hodges SJ, Davies NA, et al. Neutrophil dysfunction in alcoholic hepatitis superimposed on cirrhosis is reversible and predicts the outcome. Hepatology (2007) 46(3):831-40. doi: 10.1002/hep.21737

11. Taylor NJ, Nishtala A, Manakkat Vijay GK, Abeles RD, Auzinger G, Bernal W, et al. Circulating neutrophil dysfunction in acute liver failure. Hepatology (2013) 57(3):1142-52. doi: 10.1002/hep.26102

12. Xu R, Bao C, Huang H, Lin F, Yuan Y, Wang S, et al. Low expression of CXCR1/2 on neutrophils predicts poor survival in patients with hepatitis B virus-related acute-on-chronic liver failure. Sci Rep (2016) 6:38714. doi: $10.1038 /$ srep38714

13. Taylor NJ, Manakkat Vijay GK, Abeles RD, Auzinger G, Bernal W, Ma Y, et al. The severity of circulating neutrophil dysfunction in patients with cirrhosis is
81670567), the 13-5 State S\&T Projects of China (2018ZX10302-206) and the Fundamental Research Funds for the Central Universities.

\section{ACKNOWLEDGMENTS}

We thank all the colleagues who provide enthusiastic help for the study in State Key Laboratory for Diagnosis and Treatment of Infectious Diseases, The First Affiliated Hospital, Zhejiang University.

\section{SUPPLEMENTARY MATERIAL}

The Supplementary Material for this article can be found online at: https://www.frontiersin.org/articles/10.3389/fimmu.2021. 620365/full\#supplementary-material

associated with 90-day and 1-year mortality. Aliment Pharmacol Ther (2014) 40(6):705-15. doi: 10.1111/apt.12886

14. Gong J, Zhou W, Xiao C, Jie Y, Zhu S, Zheng J, et al. A nomogram for predicting prognostic value of inflammatory biomarkers in patients with acute-on-chronic liver failure. Clin Chim Acta (2018) 478:7-12. doi: 10.1016/ j.cca.2017.12.018

15. Gao F, Zhang Q, Liu Y, Gong G, Mao D, Gong Z, et al. Nomogram prediction of individual prognosis of patients with acute-on-chronic hepatitis B liver failure. Dig Liver Dis (2019) 51(3):425-33. doi: 10.1016/j.dld.2018.08.023

16. Wu T, Li J, Shao L, Xin J, Jiang L, Zhou Q, et al. Development of diagnostic criteria and a prognostic score for hepatitis B virus-related acute-on-chronic liver failure. Gut (2018) 67(12):2181-91. doi: 10.1136/gutjnl-2017-314641

17. Blei AT, Cordoba JG. Practice Parameters Committee of the American College of. Hepatic Encephalopathy. Am J Gastroenterol (2001) 96(7):1968-76. doi: 10.1111/j.1572-0241.2001.03964.x

18. Malinchoc M, Kamath PS, Gordon FD, Peine CJ, Rank J, ter Borg PC. A model to predict poor survival in patients undergoing transjugular intrahepatic portosystemic shunts. Hepatology (2000) 31(4):864-71. doi: 10.1053/he.2000.5852

19. Masuda S, Shimizu S, Matsuo J, Nishibata Y, Kusunoki Y, Hattanda F, et al. Measurement of NET formation in vitro and in vivo by flow cytometry. Cytometry A (2017) 91(8):822-9. doi: 10.1002/cyto.a.23169

20. Shi Y, Wu W, Yang Y, Yang Q, Song G, Wu Y, et al. Decreased Tim-3 expression is associated with functional abnormalities of monocytes in decompensated cirrhosis without overt bacterial infection. J Hepatol (2015) 63(1):60-7. doi: 10.1016/j.jhep.2015.02.020

21. Rigby KM, DeLeo FR. Neutrophils in innate host defense against Staphylococcus aureus infections. Semin Immunopathol (2012) 34(2):23759. doi: 10.1007/s00281-011-0295-3

22. Li H, Chen LY, Zhang NN, Li ST, Zeng B, Pavesi M, et al. Characteristics, Diagnosis and Prognosis of Acute-on-Chronic Liver Failure in Cirrhosis Associated to Hepatitis B. Sci Rep (2016) 6:25487. doi: 10.1038/srep25487

23. Allen L-AH, Criss AK. Cell intrinsic functions of neutrophils and their manipulation by pathogens. Curr Opin Immunol (2019) 60:124-9. doi: 10.1016/j.coi.2019.05.004

24. Brinkmann V, Reichard U, Goosmann C, Fauler B, Uhlemann Y, Weiss DS, et al. Neutrophil extracellular traps kill bacteria. Science (2004) 303 (5663):1532-5. doi: 10.1126/science.1092385

25. Manfredi AA, Ramirez GA, Rovere-Querini P, Maugeri N. The Neutrophil's Choice: Phagocytose vs Make Neutrophil Extracellular Traps. Front Immunol (2018) 9:288. doi: 10.3389/fimmu.2018.00288

26. Albillos A, Lario M, Alvarez-Mon M. Cirrhosis-associated immune dysfunction: distinctive features and clinical relevance. J Hepatol (2014) 61 (6):1385-96. doi: 10.1016/j.jhep.2014.08.010 
27. Skrzeczynska-Moncznik J, Bzowska M, Nogiec A, Sroka A, Zarebski M, Vallieres L, et al. Rapid externalization of $27-\mathrm{kDa}$ heat shock protein (HSP27) and atypical cell death in neutrophils treated with the sphingolipid analog drug FTY720. J Leukoc Biol (2015) 98(4):591-9. doi: 10.1189/jlb.3VMA1114-522RR

28. Zhang Y, Zhao R, Shi D, Sun S, Ren H, Zhao H, et al. Characterization of the circulating microbiome in acute-on-chronic liver failure associated with hepatitis B. Liver Int (2019) 39(7):1207-16. doi: 10.1111/liv.14097

29. Satsangi S, Duseja A, Sachdeva M, Tomer S, Arora SK, Taneja S, et al. Monocyte human leukocyte antigen - Antigen D related, neutrophil oxidative burst and cytokine analysis in patients of decompensated cirrhosis with and without acute-on chronic liver failure. PloS One (2018) 13(7):e0200644. doi: 10.1371/journal.pone.0200644

30. Parker LC, Whyte MK, Dower SK, Sabroe I. The expression and roles of Tolllike receptors in the biology of the human neutrophil. J Leukoc Biol (2005) 77 (6):886-92. doi: 10.1189/jlb.1104636

31. Makkar K, Tomer S, Verma N, Rathi S, Arora SK, Taneja S, et al. Neutrophil dysfunction predicts 90-day survival in patients with acute on chronic liver failure: A longitudinal case-control study. JGH Open (2020) 4(4):595-602. doi: 10.1002/jgh3.12344

32. Sharma S, Lal SB, Sachdeva M, Bhatia A, Varma N. Role of Granulocyte Colony Stimulating Factor on the Short-Term Outcome of Children with Acute on Chronic Liver Failure. J Clin Exp Hepatol (2020) 10(3):201-10. doi: 10.1016/j.jceh.2019.10.001

33. Simonetto DA, Shah VH, Kamath PS. Improving survival in ACLF: growing evidence for use of G-CSF. Hepatol Int (2017) 11(6):473-5. doi: 10.1007/ s12072-017-9834-x

34. Bober LA, Grace MJ, Pugliese-Sivo C, Rojas-Triana A, Waters T, Sullivan LM, et al. The effect of GM-CSF and G-CSF on human neutrophil function. Immunopharmacology (1995) 29(2):111-9. doi: 10.1016/0162-3109(94)00050-p

35. Clark SR, Ma AC, Tavener SA, McDonald B, Goodarzi Z, Kelly MM, et al. Platelet TLR4 activates neutrophil extracellular traps to ensnare bacteria in septic blood. Nat Med (2007) 13(4):463-9. doi: 10.1038/nm1565
36. McDonald B, Urrutia R, Yipp BG, Jenne CN, Kubes P. Intravascular neutrophil extracellular traps capture bacteria from the bloodstream during sepsis. Cell Host Microbe (2012) 12(3):324-33. doi: 10.1016/j.chom. 2012.06.011

37. Tanaka K, Koike Y, Shimura T, Okigami M, Ide S, Toiyama Y, et al. In vivo characterization of neutrophil extracellular traps in various organs of a murine sepsis model. PloS One (2014) 9(11):e111888. doi: 10.1371/journal.pone.0111888

38. Merza M, Hartman H, Rahman M, Hwaiz R, Zhang E, Renstrom E, et al. Neutrophil Extracellular Traps Induce Trypsin Activation, Inflammation, and Tissue Damage in Mice With Severe Acute Pancreatitis. Gastroenterology (2015) 149(7):1920-1931 e8. doi: 10.1053/j.gastro.2015.08.026

39. Noubouossie DF, Reeves BN, Strahl BD, Key NS. Neutrophils: back in the thrombosis spotlight. Blood (2019) 133(20):2186-97. doi: 10.1182/blood2018-10-862243

40. Nakazawa D, Shida H, Kusunoki Y, Miyoshi A, Nishio S, Tomaru U, et al. The responses of macrophages in interaction with neutrophils that undergo NETosis. J Autoimmun (2016) 67:19-28. doi: 10.1016/j.jaut.2015.08.018

41. Rodriguez-Espinosa O, Rojas-Espinosa O, Moreno-Altamirano MM, Lopez-Villegas EO, Sanchez-Garcia FJ. Metabolic requirements for neutrophil extracellular traps formation. Immunology (2015) 145(2):213-24. doi: 10.1111/imm.12437

Conflict of Interest: The authors declare that the research was conducted in the absence of any commercial or financial relationships that could be construed as a potential conflict of interest.

Copyright $\odot 2021$ Wu, Sun, Wang, Zhao, Ren, Li, Zhao, Zhang, Sheng, Chen and Shi. This is an open-access article distributed under the terms of the Creative Commons Attribution License (CC BY). The use, distribution or reproduction in other forums is permitted, provided the original author(s) and the copyright owner(s) are credited and that the original publication in this journal is cited, in accordance with accepted academic practice. No use, distribution or reproduction is permitted which does not comply with these terms. 